ISSN: 2215-2644

revedu@gmail.com

Universidad de Costa Rica

Costa Rica

\title{
Relación entre los estilos de aprendizaje y el rendimiento académico del estudiantado de la carrera de Ingeniería Agronómica de la Universidad Central del Ecuador
}

\author{
Vivas-Vivas, Ramiro José; Cabanilla-Vasconez, Enrique; Vivas Vivas, Walter Hernán \\ Relación entre los estilos de aprendizaje y el rendimiento académico del estudiantado de la carrera de Ingeniería \\ Agronómica de la Universidad Central del Ecuador \\ Revista Educación, vol. 43, núm. 1, 2019 \\ Universidad de Costa Rica, Costa Rica \\ Disponible en: http://www.redalyc.org/articulo.oa?id=44057415039 \\ DOI: https://doi.org/10.15517/revedu.v43i1.28439
}

Esta obra está bajo una Licencia Creative Commons Atribución-NoComercial-SinDerivar 3.0 Internacional. 


\title{
Relación entre los estilos de aprendizaje y el rendimiento académico del estudiantado de la carrera de Ingeniería Agronómica de la Universidad Central del Ecuador
}

\author{
The Relationship between Learning Styles and Academic Performance among Agricultural Engineering Majors at \\ the Central University of Ecuador
}

Ramiro José Vivas-Vivas

Universidad Central del Ecuador, Ecuador

DOI: https://doi.org/10.15517/revedu.v43i1.28439

rjvivas@uce.edu.ec

(iD http://orcid.org/https://

orcid.org/0000-0002-3319-8725

Enrique Cabanilla-Vasconez

Universidad Central del Ecuador, Ecuador

eacabanilla@uce.edu.ec

(iD http://orcid.org/https://

orcid.org/0000-0002-2853-291X

Walter Hernán Vivas Vivas

Universidad Técnica de Ambato, Ecuador

walter_vivasec@hotmail.com

(iD http://orcid.org/https://

orcid.org/0000-0002-4375-9557

\section{Resumen:}

El propósito de este trabajo fue determinar la influencia que tienen los estilos de aprendizaje en el rendimiento estudiantil. La investigación fue de tipo descriptivo-correlacional. El instrumento utilizado fue el cuestionario propuesto por Cabrera (2004) en su tesis doctoral, el cual fue aplicado a estudiantes del primer semestre de la carrera de Agronomía. El cálculo de la muestra se determinó con un mínimo del 95\% de confianza y un máximo de 5\% de margen de error. La población fue de 90 estudiantes, de los cuales 69 contestaron el cuestionario, lo que permitió contar con lo que se requería para la muestra. Para obtener los datos del rendimiento académico, se utilizaron las calificaciones registradas en el sistema académico de la universidad. La relación de las variables se obtuvo mediante la aplicación de la prueba de independencia/contingencia, en función de la prueba Chi Cuadrado. Los resultados de la relación entre las variables indicaron que estas son independientes, sin importar si las asignaturas pertenecen a ciencias exactas. El p, valor obtenido en todos los casos fue mayor de 0,05.

Palabras ClaVe: Aprendizaje, Rendimiento académico, Agronomía.

\section{Abstract:}

The purpose of this study is to gauge how learning styles influence academic student performance. The research method used was descriptive and correlational. The questionnaire used to assess learning styles was first proposed by Cabrera (2004) in his doctoral thesis. Agricultural Engineering students were asked to complete the survey during the first semester. The sample was calculated using a $95 \%$ confidence interval as a minimum with a $5 \%$ margin of error as a maximum. The sample population included 90 students, 69 of whom met the sample group criteria. Academic student performance was based on university records. The Chi Square Test of Independence was used to determine the relationship among the variables. The results of the variables had a significant $\mathrm{p}$ value for all cases that was higher than 0.05 , reflecting that there is independence among the courses whether they were in the Pure Sciences or not.

KEYWORDs: Learning, Academic Performance, Agricultural Engineering. 


\section{INTRODUCCIÓN}

La vida actual exige un individuo integral, que aporte a la conformación de una sociedad más equitativa y justa. En este contexto, el reto de la universidad en el pregrado debe ser la formación de sujetos capaces de convertirse en especialistas durante toda la vida (Vidal y Sanz, 2001), personas con actitudes emprendedoras e investigativas, que utilicen todos los recursos que existen en el siglo XXI, en busca de información actualizada, especializada y confiable, que le permita interiorizar el aprendizaje. Este objetivo solo se consegue si la población estudiantil hace conciencia sobre cómo aprende.

Según Purves (2001) el aprendizaje es la forma como se adquiere nuevos conocimientos. Para que éste se dé, los sistemas sensoriales tienen que ser estimulados mediante la activación de la memoria declarativa, referente a los hechos o acontecimientos, o bien la memoria de procedimiento, que incluye conductas y habilidades. Por otro lado, según Beltrán y Bueno (1995) el aprendizaje se genera cuando se produce un cambio duradero en la conducta del estudiante.

De acuerdo con Honey, Alonso y Domingo (1999) el aprendizaje se ha convertido en un proceso automático y mecánico, vinculado al estímulo-respuesta, como resultado de las situaciones que se producen en el contexto, sin embargo, se han descuidando las variables internas que favorecen la formación individuo.

En relación con el concepto de aprendizaje, según De la Torre (1993) este empieza a cuestionarse en los años 80, cuando la concepción mecánica es estimulada por situaciones externas y da paso a un modelo donde el individuo construye su aprendizaje mediante la interacción estudiante-experiencia-información, contribuyendo con la inclusión de los aspectos cognitivo, afectivo y efectivo, en el concepto de aprendizaje (De la Torre, 1993).

Esta nueva concepción del aprendizaje permite generar una línea de investigación sobre las particularidades de cada persona en el proceso de aprender, es decir, orienta el estudio hacia los estilos de aprendizaje que prevalecen en cada individuo. .

Desde la perspectiva de Vivas, Yánez y Pérez (2018) "los documentos publicados alrededor de las Ciencias Agrícolas dan énfasis a la investigación experimental, dejando relegada la investigación educativa, dando como resultado pocos estudios sobre el tema a nivel nacional e internacional" (p.36). Esta necesidad de información en materia educativa fue lo que motivó esta investigación, en la cual se considera como sujeto esencial del proceso de enseñanza-aprendizaje al estudiante y se plantean los siguientes objetivos:

Finalmente, el objetivo general gira en torno a establecer la relación existente entre estilos de aprendizaje y el rendimiento académico de los estudiantes de la carrera de Ingeniería Agronómica de la Universidad Central del Ecuador. Los objetivos específicos se centran en determinar la relación que existe entre el rendimiento académico de estos estudiantes y su estilo de aprendizaje, su forma de procesar la información, de planificar el tiempo, de comunicarse y las relaciones interpersonales que establecen.

\section{REFERENTES CONCEPTUALES}

\section{Estilos de aprendizaje}

El aprendizaje se define como un proceso que genera un cambio en la conducta y el comportamiento de un individuo, siendo el resultado de práctica o experiencia (Schunk, 2012). Esto implica que en todos los ciclos de la vida se aprende según las experiencias vividas y los conocimientos adquiridos, los cuales van a variar de una persona a otra y según las circunstancias individuales. Existen varias clasificaciones o dimensiones usadas para catalogar los estilos de aprendizaje. Desde acuerdo con Camareno, Del Buey y Herrero (2000), según la preferencia individual de adquisición del conocimiento, se dividen en el estilo activo, el reflexivo, el teórico y el pragmático, que se basan en la experiencia directa, en la observación, en la conceptualización abstracta y en la experimentación activa, respectivamente. La clasificación usada en esta investigación se fundamenta en la concepción holística y personólogica del aprendizaje, que será detallado más adelante. 
Cazau (2001) indica que cada estudiante interpreta la información de diferente manera y esto define su estilo de aprendizaje. Se puede generalizar su forma de aprender, pero no se puede categorizar en una sola manera, esto puede variar según lo que quieran aprender. Por ejemplo, uno de los estilos de aprendizaje se basa en cómo se puede percibir la información; puede ser de manera visual, verbal o cinestésico; una persona que tienen un estilo de aprendizaje visual, puede también, en ciertas ocasiones, aprender de manera cinestésica. A pesar de esto, prevalece la teoría de que cada individuo posee diferentes velocidades de aprendizaje y diferentes maneras de captar la misma información.

El reconocimiento del estilo de aprendizaje usado por el estudiante es importante para la práctica diaria educativa. El estudiante se verá más motivado a aprender cuando el docente identifique dicho estilo de aprendizaje, ya que esto le permitirá reconocer cuáles son sus fortalezas y debilidades como aprendiz (Coffield, Moseley, Hall y Ecclestone, 2004). En relación con los estudiantes universitarios, se ha determinado que los estilos de aprendizaje influyen significativamente en la elección de la carrera, con mayor énfasis en especialidades relacionadas con humanidades y con Informática, los cuales usan estilo activo y teórico, respectivamente (Camarero et al., 2000). El docente universitario puede direccionar su enseñanza al generalizar un estilo de aprendizaje, según la carrera involucrada. Esto puede guiar la metodología empleada, sin embargo, no se debe considerar como dogma.

\section{Características de los estilos de APRENDizaJe}

La preferencia individual de adquisición del conocimiento permite que existan diversos estilos de aprendizaje. En esta investigación se basan en una concepción holística y personólogica, las cuales, según Cabrera y Fariñas (2007), se definen en cuatro dimensiones: "estilos de aprendizaje relacionados con las formas preferidas de los estudiantes de percibir la información (...), de procesar la información, (...) de planificar su tiempo (...) y de orientarse hacia la comunicación y sus relaciones interpersonales en el aprendizaje" ( (p.6). Cada uno posee características relacionadas con un aprendizaje integral, caracterizando al humano como un ser complejo, que posee varias formas de abordar una misma información. Estas serán detalladas a continuación.

\section{Percibir LA INFORMACión}

Los estilos de aprendizaje que se basan en la percepción de la información se clasifican en estilos visuales, verbal/auditivo y cinético/táctil (Cabrera y Fariñas, 2007). El abordaje principal son los canales por los que se recibe el aprendizaje. Este modelo es propuesto principalmente por Dunn (1995) y Griggs (2003) (citados por Coffield et al., 2004), quienes postulan que al "adoptar técnicas específicas o hacer cambios en el entorno como la luz, el sonido, hora del día o al moverse” (p.7). Esto permite que los estudiantes aprendan según sus preferencias. Las modalidades perceptuales que se usan en este método, se conceptualizan como la adaptación al medio, según las formas preferidas del estudiante para estimular los estilos involucrados, sean de forma visual, auditiva o táctil (Cabrera y Fariñas, 2007). Sin embargo, este método tiene varias críticas, ya que no se considera un método sofisticado de aprendizaje, por su diseño y la fiabilidad de los instrumentos empleados. La aplicabilidad del método se restringe al tener que generalizar una vía de percepción de la información a todos los estudiantes, esto hace que solo los aprendices que tienen fuerte preferencia de esta vía logren el efecto deseado (Coffield et al., 2004).

Como se mencionó, es un método que se basa en preferencias, según vías de percepción de la información, conceptualmente estos estímulos generan atención e interés y permite la captación del mensaje, sin embargo, las investigaciones sobre este método muestran poco impacto así como dudosa aplicabilidad y confiabilidad. 


\section{Procesar la información}

En 1998 Riding y Rayner catalogaron a los estilos de análisis cognitivo en dos dimensiones, aquellos con vías de procesamiento de la información y con vías de representar la información (Coffield et al., 2004). En este apartado nos enfocaremos en la primera. Se distinguen dos estilos de aprendizaje basado en el procesamiento de la información, estos son: estilo global y estilo analítico.

Este modelo se fundamenta en estrategias de aprendizajes independientes de la inteligencia, es decir, su base conceptual es la manera de abordar un tema, más no su nivel de aprendizaje. Se evidencia que los aprendices holísticos o globales aprenden mejor con un generalizaciones (Coffield et al., 2004), más los aprendices analíticos procesan la información de una manera más abstracta pero de forma reflexiva y más profunda (Cabrera y Fariñas, 2007).

El método muestra debilidades en el diseño del modelo y en la validez. Por un lado, la clasificación es muy específica y se enfoca solo en aspectos cognitivos más no afectivos, por lo que algunos estudiantes no podrían ser incluidos en alguna de las dos dimensiones. Por otro lado, no hay evidencia consistente en su aplicabilidad (Coffield et al., 2004), ya que en la práctica, el maestro usa varios mecanismos de enseñanza que abarca otros estilos descritos anteriormente.

Las maneras en las que se procesa la información tienen implicaciones en el ámbito pedagógico. Por un lado, la distinción entre estudiantes con procesamiento analítico y global le da simplicidad, sin embargo, la validez y el impacto del modelo en la parte práctica lo hace inconcluso.

\section{Planificar EL TIEMPo}

En relación a los estilos de aprendizaje que se enfocan en la manera de planificar su tiempo, se reconocen al estilo planificado y al espontáneo. En primer lugar, los estudiantes que planifican el tiempo tienden a ser reflexivos y teóricos. Antes de realizar una actividad la analizan, desarrollan estrategias para su resolución y determinan el tiempo que necesitan para ejecutarla. Esto permite que auto regulen su aprendizaje y como resultado la realización de tareas eficaces planificadas, reguladas y controladas (Gravini y Iriarte, 2008).

En segundo lugar, los estudiantes que usan el estilo espontaneo suelen organizar sus ideas sin pensar demasiado. Los estudiantes que practican este estilo de aprendizaje tienden hacer las cosas más rápido, ya que no suelen gastar mucho tiempo en una misma actividad (Gravini y Iriarte, 2008). Se caracterizan por ser innovadores, por buscar actividades que les permita desarrollar su imaginación sin ninguna planificación.

Las desventajas de los estilos planificados y espontáneos se basan en el diseño del modelo y en la evaluación, respectivamente. El estilo planificado no se considera un modelo dinámico de aprendizaje, sino más bienuno inflexible (Coffield et al., 2004). El estilo espontáneo, por su parte, tiene la desventaja que durante el proceso de las tareas no se realizan retroalimentaciones y al obtener un producto final de aprendizaje si se requieren correcciones son difíciles de hacer (Gravini y Iriarte, 2008). Además, no siguen un determinado orden y no tienen una planificación concreta por lo que su nivel de reflexión es débil.

\section{COMUNICACIÓN Y RELACIONES INTERPERSONALES}

Este tipo de aprendices comunicativos adquieren sus conocimientos a través de hablar con pares o de manera individual, charlas extracurriculares y conversaciones en general en diferentes lugares y con varias personas; además usan como fuente de información revistas, televisión, audios, etcétera (Nunan, 1998). Se reconocen dos modelos según el estilo de aprendizaje asociado a la forma de comunicación y relaciones interpersonales, a saber: estilo cooperativo e independiente/individual. 
El estilo de aprendizaje cooperativo se basa en un grupo pequeño de aprendices quienes realizan varias actividades previamente explicadas con el objetivo de aprender una materia específica (Barron y Darling, 2008). Este método se ha estudiado ampliamente en meta-análisis mostrando un beneficio en el aprendizaje estadísticamente significativo en comparación con el aprendizaje individual (Johnson, Maruyuma, Johnson, Nelson, y Skon, 1981). Las principales ventajas son el mejoramiento de las relaciones sociales, la disminución del tiempo en las tareas y el comportamiento positivo hacia sus pares (Barron y Darling, 2008). Se evidencian beneficios en el aprendizaje de los estudiantes, sin embargo, este método es de difícil implementación. Se requiere un amplio conocimiento del manejo de grupos, del desarrollo de normas que permitan el trabajo en conjunto, de la planificación de tareas enfatizadas en el aprendizaje grupal y estrategias de discusión fomentando un aprendizaje significativo (Barron y Darling, 2008).

Por otro lado, el estilo de aprendizaje individual se basa en el estudio independiente, donde el estudiante puede ir a su ritmo y avanzar según su necesidad de aprendizaje. Un estudio muestra que los aprendices dotados prefieren este modelo al momento de realizar tareas en el área de matemática (Li y Adamson, 1992). Varios meta análisis han comparado el estilo individual con el cooperativo, mostrando que el trabajo en grupo no solo es superior, sino que también mejora el rendimiento al ser evaluados posteriormente de manera individual (Barron y Darling, 2008).

\section{RENDIMIENTO ACADÉMICO}

De acuerdo con Garbanzo ( 2007) el rendimiento académico es un conjunto de factores que inciden en el aprendizaje del estudiante y en el logro obtenido, sea de forma inmediata, medido por notas, o de forma mediata, vista en el desempeño profesional Los elementos involucrados se agrupan en factores tanto internos de la persona como externos considerados a los profesores y en sí a la institución (Montero, Villalobos y Valverde, 2007), tomando en cuenta al acceso de información, disponibilidad de recursos y a la infraestructura.

Entre los factores que inciden en el rendimiento académico se pueden citar: motivación, conocimientos previos, aptitudes, creencias, personalidad y estilos de aprendizaje (Cabrera y Fariñas, 2007).

La complejidad de determinar los factores más relevantes que contribuyen en el desempeño, puede excluir a otros que no por ser secundarios dejan de ser determinantes, sin embargo, los que se detallan se catalogan como los más importantes en varios artículos.

\section{EL NIVEL INTELECTUAL}

La inteligencia es uno de los factores más influyentes a la hora de predecir el rendimiento académico. Se considera dentro de las tres determinantes más relevantes, y es por ello que existen varias pruebas estandarizadas que permiten estimar el nivel de inteligencia (Edel, 2003). El nivel intelectual evidenciado en estas pruebas, permitiría a los docentes, encontrar a los estudiantes con factor de riesgo para deserción escolar si no superan el rango deseado. Hay que tomar en consideración que si bien es uno de los factores más estudiados por su importancia no necesariamente determina por si solo al desempeño académico.

El nivel intelectual se considera un factor interno de rendimiento académico, sin embargo, se encuentra ampliamente relacionado con los elementos socioculturales de su entorno familiar. Si en su ambiente externo existe un incentivo familiar y este facilita el acceso a la educación, la persona tendrá un adecuado desarrollo intelectual (Montero et al., 2007). Así, el nivel intelectual es una suma compleja de factores internos como externos. 


\section{LA PERSONALIDAD Y AUTOESTIMA}

Los rasgos de personalidad y los factores asociados a la autoestima se encuentran dentro de las variables cognitivas y emocionales (Garbanzo, 2007). La personalidad es "una organización jerárquica de sistemas, subsistemas y rasgos que transducen, transforman e integran la información", considerando sus tres elementos: la visión del mundo, los estilos de vida y la autoimagen o auto concepto (González, Núnez, Glez y García, 1997, p. 47). Las diferencias entre las personalidades de los alumnos influyen en su aprendizaje, los rasgos que más inciden son la agresividad, la ansiedad, la motivación, la autoconfianza y la timidez (Crozier, 2001). Los rasgos de la personalidad pueden ser facilitadores o inhibidores del rendimiento académico, y estos a su vez pueden o no modifican otras variables como la inteligencia (Montero et al., 2007).

La autoestima, como aspecto interno determinante en el desempeño académico, forma parte de la personalidad. Se define como "un sistema complejo y dinámico de creencias que un individuo considera verdaderas respecto a si mismo teniendo cada creencia un valor” (González et al., 1997, p. 273). La dimensión del autoestima en el rendimiento académico tiene repercusión, tanto en el éxito como en el fracaso de una persona, dependerá de cómo quiere verse y cómo quiere que los demás la perciban (Crozier, 2001; Edel, 2003).

\section{LA MOTIVACIÓN Y LOS INTERESES}

La motivación se considera una de las variables personales determinantes del desempeño escolar, es una conducta que dirige al cumplimiento de una meta y puede tener fuentes intrínsecas y extrínsecas (Edel, 2003). La motivación académica intrínseca se define "como un estado psicológico relacionado con los estudios que es positivo y significativo” (Garbanzo, 2007, p. 48) y juega un papel importante en la dedicación a sus responsabilidades como estudiante.

Por otro lado, la motivación extrínseca se enfatiza en el apoyo obtenido de fuentes externas; el ambiente académico, los servicios ofrecidos por la universidad, los factores socioeconómicos, entre otros; su interacción afectapositiva o negativamente en el desempeño académico (Garbanzo, 2007). Los padres, los docentes, los compañeros y personas cercanas tienen un rol relevante, ya que su apoyo continuo respalda tanto afectiva como académicamente al estudiante, al plantearse una meta Es así que motivar a los estudiantes implica fomentar sus recursos internos, su sentido de competencia, autoestima, autonomía y realización" (Edel, 2003, p. 5). El nivel de motivación se relaciona proporcionalmente con el interés que pone en sus actividades, así como las aspiraciones (Montero et al., 2007). Cuando hay interés en la realización de una actividad, el estudiante se encuentra motivado, no se detiene a pensar en el tiempo o el camino que necesitaría para lograr la meta planteada, sino más bien se concentra en cumplir con su objetivo y terminar lo iniciado.

\section{LAS APTITUDES}

Las aptitudes se encuentran dentro de las variables personales que condicionan el rendimiento académico junto con la inteligencia (González et al., 1997). Se definen como "habilidades para realizar determinadas tareas por parte del estudiante, mediante diferentes pruebas” (Garbanzo, 2007, p. 52).

\section{HÁBitos de ESTUdio}

Los hábitos de estudio son las costumbres usadas para aprender, es decir, "la forma en que el individuo se organiza en cuanto a tiempo, espacio, técnicas y métodos concretos que utiliza para estudiar" (Cartagena, 2008, p. 65). 
Los hábitos de estudio se van formando a lo largo de la vida académica y según la priorización que se ponga al estudio, determinará la relevancia de invertir más tiempo o de usar técnicas efectivas de aprendizaje. Esta rutina se va adquiriendo según las influencias recibidas como consecuencias al realizar una determinada tarea. La metodología de enseñanza y la evaluación usada por el docente al momento de revisar una tarea, refuerza algunas conductas de estudio, es decir, los hábitos se fortalecen "en función al número de refuerzos que las conductas hayan recibido" sean positivas o negativas (Cartagena, 2008, p.65). Si un estudiante muestra poco esfuerzo y dedicación al realizar una tarea escolar, y el docente, por su lado, la evalúa de forma positiva, se va formando inadecuados hábitos de estudio, ya que el aprendiz aplicará poco tiempo, no realizará buenas técnicas de estudio y sus rutinas van adquiriendo una tendencia negativa.

Esta variable es determinante en el rendimiento académico del estudiante, ya que se va formando desde la infancia, se refuerza en la adolescencia y se aplica de forma inconsciente en todas las situaciones de la vida.

\section{INDICADORES DEL RENDIMIENTO ACADÉMICO}

Para valorar el rendimiento académico se usan indicadores, que son variables que nos permiten determinar tanto de forma mediata como inmediata el desempeño escolar alcanzado. La forma más usada son las calificaciones que son la valoración objetiva o subjetiva del docente mostrada en números o letras a 1 trabajo realizado por el estudiante. Sin embargo, este criterio es problemático al enfocarnos en el rendimiento académico universitario, es por eso que la mejor forma de valorarlo es analizando la tasa de éxito, tasa de retraso y abandono del estudiante de educación superior (Garbanzo, 2007).

Hay que tomar en consideración que estos indicadores son el conjunto de factores sociodemográficos y personales que interaccionan entre sí para contribuir en del desempeño escolar de un individuo.

\section{LA TASA DE ÉXITO}

El éxito en el ámbito escolar depende de varios factores que impulsa positivamente al desenvolvimiento académico. La autoestima y la motivación que tengan en aprender y estudiar son los principales factores que influyen para lograr el éxito (Román, 2013). Los docentes, por su parte, tienen un rol importante en la motivación que tengan los estudiantes, así como en el interés. Los estudiantes al "ser percibidos como hábiles y capaces, otorga bases fundadas para el desarrollo de su autoestima, creándose así un círculo positivo de profecía de éxito" (Román, 2013, p. 50).

Los estudiantes con alta motivación y confianza en sí mismos son individuos con alta tasa de éxito escolar (Edel, 2003). El esfuerzo en la realización de tareas, el cumplimiento de sus obligaciones y la capacidad de ordenar sus prioridades son características para que el aprendiz logre sus metas. La valoración de éxito se realiza analizando los logros personales y profesionales, cuyo resultado es lo que se logra desde el punto de vista del aprendizaje (Garbanzo, 2007).

\section{LA TASA DE REPITENCIA}

La tasa de repitencia se define, según el Instituto Nacional de Estadística y Censos del Ecuador, como el "número de personas que se han matriculado más de una vez en el mismo grado o nivel en educación escolarizada ordinaria" en un periodo específico (Ministerio de Educación del Ecuador, 2013, p.33). Según el último estudio realizado por el Instituto Nacional de Estadística y Censos (2012), en el año 2012, el porcentaje de repitencia en la Educación Media es mayor que en la Educación Superior, correspondiendo a 6,9\% y 4,4\% respectivamente en el año 2010. El Consejo de Evaluación, Acreditación y Aseguramiento de 
la Calidad de la Educación (CEAACES) no tienen datos de las universidades ecuatorianas sobre la tasa de repitencia y tan solo posee datos sobre el índice de retención y titulación.

La tasa de repitencia aumenta el riesgo de deserción escolar y es más común en zonas rurales con bajos niveles socioeconómicos (UNESCO, 2009). Se correlaciona de forma inversa con el rendimiento académico. Existen varios factores que afectan, ya sea de forma endógena o exógena y, estos se encuentran asociados a la deserción escolar, tema que se aborda en el siguiente apartado.

\section{LA TASA DE DESERCIÓN}

La deserción escolar es la separación del estudiante del entorno educativo al que estuvo inmerso, por factores ya sean internos o externos. Por un lado, los factores internos asociados a la desvinculación definitiva de la educación escolar son: repeticiones reiteradas, motivaciones bajas, relacionados con la edad (mayores en relación a sus compañeros), falta de apoyo, falta de confianza, discriminación, entre otros (Román, 2013).

Por otro lado, se ha determinado que el nivel de educación promedio de los hogares y el nivel socioeconómico promedio de los alumnos, como factores externos, influyen de forma inversamente proporcional a la probabilidad de abandono escolar (UNESCO, 2009). Otros factores externos son: trabajo juvenil, estructura familiar, contexto social (mayor en zonas rurales), maternidad o paternidad temprana y salida temprana del hogar (Román, 2013). Para atender estas temáticas hay programas de reinserción, así como estrategias de prevención, en estudiantes con alto riesgo de deserción escolar, en varios países.

El Banco Mundial (2017) menciona, que de los estudiantes que ingresan a la universidad solo el $50 \%$ llegan a obtener su título universitario. En lo referente al Ecuador el porcentaje de deserción estudiantil es del 26\% (SENESCYT, 2017).

\section{Procedimientos metodológicos}

Los estudiantes que ingresaron a la carrera de Ingeniería Agronómica en el semestre de septiembre del 2015 a febrero del 2016, tomaron obligatoriamente las asignaturas de Química Aplicada, Biología Aplicada, Métodos de Estudio, Introducción a la Agronomía, Cálculo Diferencial y Física Aplicada. Se matricularon 90, divididos en cuatro paralelos. Para el cálculo de la muestra se consideró un margen de error máximo de $5 \%$ y un nivel mínimo de confianza de $95 \%$.

El propósito de la investigación fue determinar la influencia que ejerce los estilos de aprendizaje en el rendimiento estudiantil, por esta razón, el tipo de investigación fue descriptivo-correlacional. En primer lugar se midieron las variables, seguidamente se analizó la correlación existente entre estas (Hernández-Díaz, 2005).

Para la adquisición de la información se utilizó el cuestionario propuesto por Cabrera (2004). Este instrumento toma en consideración las formas de percibir, procesar, planificar y comunicar que usa el estudiante para desarrollar su proceso de aprendizaje. Este cuestionario fue tipo Likert, donde el estudiante tenía las opciones de escoger entre las posibilidades: totalmente de acuerdo, a veces, no lo sé y nunca. El utilitario Google Drive fue donde se publicó el instrumento y los estudiantes accedieron a este en su tiempo libre con la finalidad de realizar la actividad. 69 estudiantes respondieron al cuestionario, 37 hombres y 32 mujeres, con edades que fluctúan entre los 18 y 20 años. Sin embargo, en este estudio no se consideraron las características de género ni de edad.

El tiempo que toma para la resolución del instrumento es pequeño, nunca mayor a los 20 minutos. Los criterios que se usaron para el análisis de los resultados sobre los estilos de aprendizaje, fueron los propuestos por el autor del cuestionario y se los puede observar en la Tabla 1. 
TABLA 1

Criterios de evaluación de los estilos de aprendizaje

\begin{tabular}{|l|l|l|}
\hline Estilo & Canal & Ítems \\
\hline \multirow{3}{*}{ Percibir la información } & Visual & $1,3,5$ \\
\cline { 2 - 3 } & Verbal & $2,7,8$ \\
\cline { 2 - 3 } & Cinético & $4,6,9$ \\
\hline \multirow{2}{*}{ Procesar la información } & Global & $10,13,14$ \\
\hline \multirow{2}{*}{ Planificar el tiempo } & Analítico & $11,12,15$ \\
\hline \multirow{2}{*}{$\begin{array}{c}\text { Comunicación y relaciones } \\
\text { interpersonales }\end{array}$} & Planificado & $16,19,20$ \\
\hline & Espontáneo & $17,18,20$ \\
\hline & Cooperativo & $22,24,26$ \\
\hline & Independiente & $23,25,27$ \\
\hline
\end{tabular}

Cabrera (2004)

El puntaje obtenido por cada uno de los estudiantes al finalizar el semestre y registrado en el Sistema Académico de la universidad ( $\mathrm{SiAc}$ ) por parte de los docentes, fue considerado como medida del rendimiento estudiantil.

Esta información se obtuvo de la base de datos de la Facultad de Ciencias Agrícolas. La escala para el análisis del rendimiento estudiantil fue: insatisfactorio (1-27), satisfactorio (20-31), competente (32-35) y destacado (26-40).

El almacenamiento, depuración y tratamiento de la información para obtener las relaciones entre las variables, se realizó en el programa Excel. Con la finalidad de ver la relación existente entre los estilos de aprendizaje y el rendimiento estudiantil se aplicó la prueba de independencia/contingencia, en función de la prueba del chi cuadrado.

\section{ANÁLISIS Y DISCUSIÓN DE RESULTADOS}

Los resultados obtenidos de la investigación sobre la relación existente entre los estilos de aprendizaje y el rendimiento académico, indican que las dos variables son totalmente independientes.

TABLA 2

Relación de los estilos de aprendizaje y el rendimiento académico (Física y Cálculo Diferencial)

\begin{tabular}{|c|c|c|c|c|c|c|c|c|c|c|}
\hline \multirow{3}{*}{\begin{tabular}{|l} 
Estilos de \\
aprendizaje
\end{tabular}} & Tipos & \multicolumn{9}{|c|}{ Niveles de rendimiento académico } \\
\hline & & \multicolumn{2}{|c|}{ Destacado } & \multicolumn{2}{|c|}{ Competente } & \multicolumn{2}{|c|}{ Satisfactorio } & \multicolumn{2}{|c|}{ Insatisfactorio } & \multirow[t]{2}{*}{ p-value } \\
\hline & & $\mathrm{F}$ & $\%$ & $\mathrm{~F}$ & $\%$ & $\mathrm{~F}$ & $\%$ & $\mathrm{f}$ & $\%$ & \\
\hline \multirow{6}{*}{$\begin{array}{l}\text { Percibir la } \\
\text { información }\end{array}$} & Visual & 3 & 4 & 1 & 1 & 21 & 30 & 7 & 10 & \multirow[t]{6}{*}{0,8246} \\
\hline & Verbal & 0 & 0 & 1 & 1 & 4 & 6 & 2 & 3 & \\
\hline & Cinético & 0 & 0 & 0 & 0 & 7 & 10 & 2 & 3 & \\
\hline & Visual-Verbal & 1 & 1 & 0 & 0 & 6 & 9 & 0 & 0 & \\
\hline & Visual-Cinético & 0 & 0 & 0 & 0 & 7 & 10 & 3 & 4 & \\
\hline & Verbal-Cinético & 0 & 0 & 0 & 0 & 3 & 4 & 1 & 1 & \\
\hline \multirow{3}{*}{$\begin{array}{l}\text { Procesar la } \\
\text { información }\end{array}$} & Global & 2 & 3 & 1 & 1 & 24 & 35 & 9 & 13 & \multirow[t]{3}{*}{0,5253} \\
\hline & Analítico & 0 & 0 & 0 & 0 & 15 & 22 & 4 & 6 & \\
\hline & Global-Analítico & 2 & 3 & 1 & 1 & 9 & 13 & 2 & 3 & \\
\hline \multirow{3}{*}{$\begin{array}{l}\text { Planificar el } \\
\text { tiempo }\end{array}$} & Planificado & 3 & 4 & 1 & 1 & 26 & 38 & 5 & 7 & \multirow[t]{3}{*}{0,3748} \\
\hline & Espontáneo & 1 & 1 & 1 & 1 & 14 & 20 & 9 & 13 & \\
\hline & Planificado-Espontáneo & 0 & 0 & 0 & 0 & 8 & 12 & 1 & 1 & \\
\hline \multirow{3}{*}{$\begin{array}{l}\text { Comunicación y } \\
\text { relaciones } \\
\text { interpersonales }\end{array}$} & Cooperativo & 2 & 3 & 0 & 0 & 21 & 30 & 6 & 9 & \multirow[t]{3}{*}{0,1015} \\
\hline & Independiente & 1 & 1 & 0 & 0 & 17 & 25 & 8 & 12 & \\
\hline & Cooperativo-Independiente & 1 & 1 & 2 & 3 & 10 & 14 & 1 & 1 & \\
\hline
\end{tabular}


En la Tabla 2 se puede observar que la relación entre los estilos de aprendizaje y el rendimiento académico de los estudiantes en las ciencias exactas conformadas por las asignaturas de Física y Cálculo Diferencial es no significativa, porque el resultado de la relación entre la forma de percibir la información y el rendimiento académico da un valor de 0,8246, que es superior a 0,05. Lo mismo sucede con las formas de procesar la información (0,5253), planificar el tiempo (0,3748). En el caso de la relación entre la forma de comunicación y las relaciones interpersonales con el rendimiento académico existe una ligera aproximación al 0,10 , lo que da un leve indicio de la influencia que tiene esta forma sobre el rendimiento.

TABLA 3

Relación de los estilos de aprendizaje y el rendimiento académico (Química y Biología)

\begin{tabular}{|c|c|c|c|c|c|c|c|c|c|c|}
\hline \multirow{3}{*}{$\begin{array}{l}\text { Estilos de } \\
\text { aprendizaje }\end{array}$} & \multirow{3}{*}{ Tipos } & \multicolumn{9}{|c|}{ Niveles de rendimiento académico } \\
\hline & & \multicolumn{2}{|c|}{ Destacado } & \multicolumn{2}{|c|}{ Competente } & \multicolumn{2}{|c|}{ Satisfactorio } & \multicolumn{2}{|c|}{ Insatisfactorio } & \multirow[t]{2}{*}{ p-value } \\
\hline & & $\mathrm{f}$ & $\%$ & $\mathrm{~F}$ & $\%$ & f & $\%$ & f & $\%$ & \\
\hline \multirow{6}{*}{$\begin{array}{l}\text { Percibir la } \\
\text { información }\end{array}$} & Visual & 0 & 0 & 0 & 0 & 12 & 17 & 20 & 29 & \multirow[t]{6}{*}{0,2468} \\
\hline & Verbal & 0 & 0 & 0 & 0 & 2 & 3 & 5 & 7 & \\
\hline & Cinético & 0 & 0 & 1 & 1 & 4 & 6 & 4 & 6 & \\
\hline & Visual-Verbal & 0 & 0 & 0 & 0 & 5 & 7 & 2 & 3 & \\
\hline & Visual-Cinético & 0 & 0 & 0 & 0 & 6 & 9 & 4 & 6 & \\
\hline & Verbal-Cinético & 0 & 0 & 0 & 0 & 3 & 4 & 1 & 1 & \\
\hline \multirow{3}{*}{$\begin{array}{l}\text { Procesar la } \\
\text { información }\end{array}$} & Global & 0 & 0 & 0 & 0 & 17 & 25 & 19 & 28 & \multirow[t]{3}{*}{0,2126} \\
\hline & Analítico & 0 & 0 & 0 & 0 & 7 & 10 & 12 & 17 & \\
\hline & Global-Analítico & 0 & 0 & 1 & 1 & 8 & 12 & 5 & 7 & \\
\hline \multirow{3}{*}{$\begin{array}{l}\text { Planificar el } \\
\text { tiempo }\end{array}$} & Planificado & 0 & 0 & 0 & 0 & 19 & 28 & 16 & 23 & \multirow[t]{3}{*}{0,3352} \\
\hline & Espontáneo & 0 & 0 & 1 & 1 & 8 & 12 & 16 & 23 & \\
\hline & Planificado-Espontáneo & 0 & 0 & 0 & 0 & 5 & 7 & 4 & 6 & \\
\hline \multirow{3}{*}{$\begin{array}{l}\text { Comunicación } \\
\text { y relaciones } \\
\text { interpersonales }\end{array}$} & Cooperativo & 0 & 0 & 0 & 0 & 14 & 20 & 15 & 22 & \multirow[t]{3}{*}{0,1208} \\
\hline & Independiente & 0 & 0 & 1 & 1 & 8 & 12 & 17 & 25 & \\
\hline & Cooperativo-Independiente & 0 & 0 & 0 & 0 & 10 & 14 & 4 & 6 & \\
\hline
\end{tabular}

Elaboración propia

En la Tabla 3 se puede observar que la relación entre los estilos de aprendizaje y el rendimiento académico de los estudiantes en las ciencias de la vida conformadas por las asignaturas de Química y Biología es no significativa, porque el resultado de la relación entre la forma de percibir la información y el rendimiento académico da un valor de 0,2468, que es superior a 0,05. Lo mismo sucede con las formas de procesar la información (0,2126), planificar el tiempo (0,3352). En el caso de la relación entre la forma de comunicación y las relaciones interpersonal con el rendimiento académico existe una ligera aproximación al 0,12 , lo que da un leve indicio de la influencia que tiene esta forma sobre el rendimiento, coincidiendo con lo observado con en la Tabla 2. 
TABLA 4

Relación de los estilos de aprendizaje y el rendimiento académico (Introducción a la Agronomía y Métodos de Estudio)

\begin{tabular}{|c|c|c|c|c|c|c|c|c|c|c|}
\hline \multirow{3}{*}{$\begin{array}{l}\text { Estilos de } \\
\text { aprendizaje }\end{array}$} & \multirow{3}{*}{ Tipos } & \multicolumn{9}{|c|}{ Niveles de rendimiento académico } \\
\hline & & \multicolumn{2}{|c|}{ Destacado } & \multicolumn{2}{|c|}{ Competente } & \multicolumn{2}{|c|}{ Satisfactorio } & \multicolumn{2}{|c|}{ Insatisfactorio } & \multirow[t]{2}{*}{ p-value } \\
\hline & & $\mathrm{f}$ & $\%$ & $\mathrm{f}$ & $\%$ & $\mathrm{f}$ & $\%$ & f & $\%$ & \\
\hline \multirow{6}{*}{$\begin{array}{l}\text { Percibir la } \\
\text { información }\end{array}$} & Visual & 1 & 1 & 1 & 1 & 26 & 38 & 4 & 6 & \multirow[t]{6}{*}{0,9398} \\
\hline & Verbal & 0 & 0 & 1 & 1 & 5 & 7 & 1 & 1 & \\
\hline & Cinético & 0 & 0 & 0 & 0 & 7 & 10 & 2 & 3 & \\
\hline & Visual-Verbal & 0 & 0 & 0 & 0 & 7 & 10 & 0 & 0 & \\
\hline & Visual-Cinético & 0 & 0 & 0 & 0 & 8 & 12 & 2 & 3 & \\
\hline & Verbal-Cinético & 0 & 0 & 0 & 0 & 3 & 4 & 1 & 1 & \\
\hline \multirow{3}{*}{$\begin{array}{l}\text { Procesar la } \\
\text { información }\end{array}$} & Global & 0 & 0 & 2 & 3 & 28 & 41 & 6 & 9 & \multirow[t]{3}{*}{0,3664} \\
\hline & Analítico & 0 & 0 & 0 & 0 & 16 & 23 & 3 & 4 & \\
\hline & Global-Analítico & 1 & 1 & 0 & 0 & 12 & 17 & 1 & 1 & \\
\hline \multirow{3}{*}{$\begin{array}{l}\text { Planificar el } \\
\text { tiempo }\end{array}$} & Planificado & 1 & 1 & 2 & 3 & 29 & 42 & 3 & 4 & \multirow[t]{3}{*}{0,4752} \\
\hline & Espontáneo & 0 & 0 & 0 & 0 & 19 & 28 & 6 & 9 & \\
\hline & Planificado-Espontáneo & 0 & 0 & 0 & 0 & 8 & 12 & 1 & 1 & \\
\hline \multirow{3}{*}{$\begin{array}{l}\text { Comunicación } \\
\text { y relaciones } \\
\text { interpersonales }\end{array}$} & Cooperativo & 1 & 1 & 1 & 1 & 23 & 33 & 4 & 6 & \multirow[t]{3}{*}{0,6745} \\
\hline & Independiente & 0 & 0 & 0 & 0 & 21 & 30 & 5 & 7 & \\
\hline & Cooperativo-Independiente & 0 & 0 & 1 & 1 & 12 & 17 & 1 & 1 & \\
\hline
\end{tabular}

\section{Elaboración propia}

En la Tabla 4 se puede observar que la relación entre los estilos de aprendizaje y el rendimiento académico de los estudiantes en las ciencias sociales conformadas por las asignaturas de Introducción a la Agronomía y Métodos de Estudio es no significativa, porque el resultado de la relación entre la forma de percibir la información y el rendimiento académico da un valor de 0,9398 que es superior a 0,05. Lo mismo sucede con las formas de procesar la información $(0,3664)$, planificar el tiempo $(0,4752)$ y se repite en la relación entre la forma de comunicación y las relaciones interpersonal $(0,6745)$.

En términos generales, se pudo constatar que la relación que existe entre el estilo percibir la información y el rendimiento académico en estudiantes de la Carrera de Agronomía de la Facultad de Ciencias Agrícolas de la Universidad central del Ecuador fue mayormente positivo. Para el caso de Introducción a la Agronomía y Métodos de Estudio la relación fue de $84 \%$, por lo que son las materias con el indicador más alto. Para el segundo grupo de materias de Física y Cálculo la relación positiva fue de un $76 \%$. Finalmente para el caso de Química y Biología hubo un comportamiento diferente, pues en esta caso tal solo el $47 \%$ de los estudiantes mostraron una relación positiva. En promedio se puede resumir que uno de cada 2 estudiantes tuvo una relación favorable en todas las materias.

Por otro lado, se evidenció que el principal tipo de relación positiva para percibir la información comparada con el rendimiento académico fue la visual, ocupando en segundo lugar el tipo visual cinético y finalmente con el mismo puntaje el tipo cinético y visual-verbal.

En segundo lugar, se evidenció que la relación que existe entre el estilo procesar la información y el rendimiento académico fue mayormente positiva, en cuatro de las seis materias evaluadas. En primer lugar, las materias de Introducción a la Agronomía y Métodos de Estudio tuvieron la mejor relación positiva con un $85 \%$. En segundo lugar, las materias de Física y Cálculo también mostraron un comportamiento positivo con un $78 \%$. Finalmente, con un porcentaje negativo, Química y Biología, tan solo mostraron un 48\% de efectividad en esta relación.

El estilo de procesamiento de información que tuvo mayor impacto positivo con el rendimiento académico de los estudiantes fue el Global, duplicando en efectividad a los estilos: Analítico y Global-Analítico

En tercer lugar, al establecer la relación que existe entre el estilo planificar el tiempo y el rendimiento académico de los estudiantes evaluados se evidenció que esta fue la relación (mayormente positiva) fue la 
más alta de entre los diferentes estilos de aprendizaje evaluados. Nuevamente las materias de Introducción a la Agronomía y Métodos de Estudio tuvieron el mayor porcentaje positivo con un $86 \%$. En segundo lugar, las materias de Física y Cálculo, también tuvieron un excelente porcentaje con un $77 \%$ de relación entre la planificación del tiempo y su rendimiento académico. Finalmente, las materias de Química y Biología, mantuvieron un resultado medio de apenas un $48 \%$ de efectividad.

El tipo planificado fue la característica que mayor efectividad tuvo en esta relación, superando por el doble a espontaneo y por cuatro veces a lo planificado-espontáneo.

Finalmente, en cuanto a la relación que existe entre el estilo comunicación y relaciones interpersonales y el rendimiento académico la evaluación fue nuevamente positiva para Introducción a la Agronomía y Métodos de Estudio con un $83 \%$ y para Física y Cálculo con un $77 \%$. Se mantiene la constante de Química y Biología donde el factor positivo apenas fue de $47 \%$.

El tipo cooperativo fue el mayormente positivo, pero sin una brecha considerable de las otras dos alternativas: Independiente y cooperativo independiente.

\section{Conclusiones}

En la forma como perciben la información los estudiantes, lo que predomina es el visual (ciencias exactas $45 \%$, ciencias de la vida $46 \%$ y ciencias humanas $46 \%$ ), no se aprecia diferencias significativas entre las formas de captar la información por medio del lenguaje, cinético y los modelos combinados. Se deduce que a pesar de tener preferencia por lo visual los estudiantes utilizan varios tipos de estilos de percibir la información al momento de aprender. En cuanto a la relación existente entre la forma de percibir la información y el rendimiento académico esta es independiente, como lo demuestra el p valor (ciencias exactas 0,8246 , ciencias de la vida 0,2468 y ciencias humanas 0,9398).

Predomina el factor global (ciencia exactas 52\%, ciencias de la vida 53\% y ciencias humanas 53\%) en la forma como el estudiante procesa la información, no se aprecia diferencias significativas entre la forma analítica y combinado. La relación existente con el rendimiento académico es no significativa (ciencias exactas 0,5253 , ciencias de la vida 0,2126 y ciencias humanas 0,3664 ).

En lo que respecta a la planificación del tiempo en el proceso de aprendizaje, los estudiantes tienen una predilección por la planificación de las actividades (ciencias exactas $50 \%$, ciencias de la vida $51 \%$ y ciencias humanas 50\%), sin embargo la forma espontánea tiene una cantidad representativa de adeptos que va entre el 36 y 37\%. Con relación a la influencia que tiene este aspecto en el rendimiento académico se vuelve a repetir el patrón anterior (relación independiente), ya que ninguna de las tres ciencias tiene un p valor menor a 0,05.

La sociabilidad en el entorno educativo para realizar las actividades académicas destinadas al aprendizaje, que ejercen los estudiantes tiende a ser cooperativa (de $41 \%$ a 42\%), no obstante el factor independiente tiene un alto grado de aceptación (de 37 a 38\%). Esto deriva en que los estudiantes no tienen una real inclinación cuando a la comunicación y relaciones interpersonales se refiere. El p valor obtenido en las tres ciencias es superior a 0,05 lo que demuestra que la relación que existe entre este factor y el rendimiento académico es no significativa.

Pese a que el rendimiento académico es independiente a los estilos de aprendizaje, se observa que este rendimiento es apenas satisfactorio (ciencias exactas 48, ciencias de la vida 32 y ciencias humanas 56, de 69 estudiantes participantes), lo que demuestra que la calidad de la educación superior debe ser fortalecida

\section{ReFERENCIAS}

Banco Mundial. (17 de mayo de 2017). Graduarse: solo la mitad lo logra en América Latina. Recuperado de https:/ /goo.gl/NbFSnh 
Barron, B. y Darling, L. (2008). Teaching for meaningful learning. A review of research on inquiry-based and cooperative learning. San Francisco, EEUU: John Wiley y Sons Inc

Beltrán, J. y Bueno, J.A. (1995). Psicología de la Educación. Baarcelona: Editorial Boixareu Univertsitaria

Cabrera, A. (2004). Fundamentos de un sistema didáctico del inglés con fines especificos centrado en los estilos de aprendizaje. (Tesis Doctoral). Universidad de Pinar del Río, Pinar del Río, Cuba.

Cabrera, J., y Fariñas, G. (2007). El estudio de los estilos de aprendizaje desde una perspectiva vigostkiana: una aproximación conceptual. Revista Iberoamericana de Educación, 37(1), 1-8.

Camarero, F., Del Buey, F. y Herrero, J. (2000). Estilos y estrategias de aprendizaje en estudiantes universitarios. Psicothema, 12(4), 615-622.

Cartagena, M. (2008). Relación entre la autoeficiencia y el rendimiento escolar y los hábitos de estudio en alumnos de secundaria. Revista Iberoamericana sobre Calidad, Eficacia y Cambio en Educación, 6(3), 60-89.

Cazau, P. (2001). Estilos de aprendizaje: Generalidades. Recuperado de https://bit.ly/2S5ZSIG

Coffield, F., Moseley, D., Hall, E. y Ecclestone, K. (2004). Should we be using learning styles? What research has to say to practice. Learning and Skills Research Centre, 1-62.

Crozier, R. (2001). Diferencias individuales en el aprendizaje-Personalidad y rendimiento escolar. Madrid: Narcea, S.A. de Ediciones.

De la Torre, S. (1993). Aprender de los errores: El tratamiento didáctico de los errores como estrategia de innovación. Argentina: Magisterio de la Plata.

Edel, R. (2003). El rendimiento académico: Concepto, Investigación y desarrollo. Revista Electrónica Iberoamericana sobre Calidad, Eficacia y Cambio en Educación, 1(2), 1-15.

Garbanzo, G. (2007). Factores asociados al rendimiento académico en estudiantes universitarios, una reflexión desde la calidad de la educación superior pública. Revista Educación, 31(1), 43-63.

González, J., Núnez, C., Glez, S. y García, M. (1997). Autoconcepto, autoestima y aprendizaje escolar. Psicothema, 9(2), 271-289.

Gravini, M. y Iriarte, F. (2008). Metacognitive processes of students with different learning styles. Psicología desde el Caribe, 22.

Hernández-Díaz, A. (2005). Las estrategias de aprendizaje como un medio de apoyo en el proceso de asimilación. (Tesis Doctoral). Universidad de la Habana, Cuba. Recuperado de: http://www.cepes.uh.cu/bibliomaestria/estrategi ascomomedio.doc

Honey, P., Alonso, C. y Domingo, J. (1994). Los estilos de aprendizaje: procedimientos de diagnóstico y mejora. España, Bilbao: Ediciones El Mensajero.

Instituto Nacional de Estadística y Censos. (2012). Análisis: Revista Coyuntural. Quito, Ecuador: e-Análisis.

Johnson, D., Maruyuma, G., Johnson, R., Nelson, D. y Skon, L. (1981). Effects of cooperative, competitive, and individulistic goal structures on achievement: A meta-analysis. Psychologycal Bulletin, 89(1), 47-62.

Li, A. y Adamson, G. (1992). Gifted Secondary Students' Preferred Learning Style: Cooperative, Competitive, or Individualistic? Journal for the Education of the Gifted, 16(1), 46-54.

Ministerio de Educación del Ecuador. (2013). Ecuador: Indicadores educativos 2011-2012. Quito: MinEduc.

Montero, E., Villalobos, P. y Valverde, A. (2007). Factores institucionales, pedagógicos, psicosociales y sociodemográficos asociados al rendimiento académico en la Universidad de Costa Rica: Un análisis multinivel. Revista Electrónica de Investigación y Evaluación Educativa, 13(2), 215-234.

Nunan, D. (1998). Language teaching methodology-A textbook for teachers. New York: Prentice-Hall International Edition.

Purves, D. (2001). Invitación a la Neurociencia. Colombia: Editorial Médica Panamericana

Román, M. (2013). Factores asociados al abandono y la deserción escolar en América Latina: Una mirada en conjunto. Revista Iberoamericana sobre Calidad, Eficacia y Cambio en Educación, 11(2), 34-54.

Schunk, D. (2012). Teorias del aprendizaje-Una perspectiva educativa. México: Pearson Educación. 
Ramiro José Vivas-Vivas, et al. Relación entre los estilos de aprendizaje y el Rendimiento académi...

SENESCYT. (23 de mayo de 2017). Informe rendición de cuentas Senescyt 2016. Recuperado de http://www.senescy t.gob.ec/rendicion2016/

UNESCO. (2009). Abandono escolar y politicas de inclusión en la educación secundaria. Buenos Aires, Argentina: PNUD

Vidal, G. y Sanz, T. (2001). La asignatura: ¿Conjunto o sistema? Revista Cubana de Educación Superior, 4(3).

Vivas, R., Yánez, S. y Pérez, J. (2018) Perfil de ingreso de los estudiantes a la facultad de Ciencias Agrícolas en la Universidad Central de Ecuador. Sophia, 14(2), 35-45.

\section{BY-NC-ND}

